\title{
Elastic jellium sphere in a static electric field
}

\author{
Ping Sheng \\ Corporate Research Science Laboratories, Exxon Research and Engineering Company, \\ Clinton Township, Annandale, New Jersey 08801
}

M. Y. Chou ${ }^{*}$ and Marvin L. Cohen

Department of Physics, University of California, Berkeley, California 94720

and Materials and Molecular Research Division, Lawrence Berkeley Laboratory, University of California,

Berkeley, California 94720

(Received 23 October 1985)

\begin{abstract}
The electronic and ionic response of a small metal particle in a static electric field is studied within the jellium model in which the ionic jellium background is assumed to be elastically deformable. Results of the calculation indicate that while the polarizability of the particle remains remarkably constant as the jellium stiffness is relaxed from perfect rigidity, there are nevertheless significant departures from the rigid jellium model in both the ionic and/or electronic makeup of the polarizability and the associated charge-density modulations. In particular, for $\mathrm{Na}$ clusters of up to 92 atoms the ionic component is shown to account for $\sim 10-15 \%$ of the total polarizability. Numerically evaluated charge-modulation profiles, together with the variation of ionic polarizability with elastic stiffness, are presented and discussed.
\end{abstract}

\section{INTRODUCTION}

Small metal clusters constitute an interesting material system which displays many novel characteristics unique to matter intermediate between a single molecule and the bulk. ${ }^{1-4}$ Recently, the success enjoyed by the jellium model in explaining the electronic shell structure observed in alkali metal clusters ${ }^{3}$ indicates that the picture of free electrons moving in a homogeneous positive background provides a reasonable physical model for understanding the electronic properties of these clusters. However, one aspect of the jellium model, i.e., the implicit assumption of jellium rigidity, is noted to depart significantly from the elastic nature of a real solid. It is the purpose of this note to examine the effect of relaxing the jellium rigidity on the static electric polarizability of a jellium sphere. Our results show that while the particle polarizability acquires an elastic (ionic) component in addition to the usual electronic component as the jellium stiffness decreases, its net value, given by the difference of the two components, increases only $1-2 \%$. The relaxation of the rigid jellium therefore does not alter the total polarizability to any significant extent. In particular, it does not increase the polarizability to the level measured experimentally (on alkali-metal-atom clusters). ${ }^{1}$ However, our calculation does indicate sizable departures from the rigid jellium model in both the ionic and/or electronic makeup of the polarizability and the associated charge density modulations. For jellium spheres with physical parameters corresponding to $\mathrm{Na}$ clusters of up to 92 atoms, the ionic component is shown to account for $10-15 \%$ of the total polarizability. In what ensues, formulation of our approach is given in Sec. II. Section III presents the numerical results together with the relevant discussion. Some mathematical details and derivations are to be found in the two appendices.

\section{FORMULATION}

Consider a sphere of radius $R$ with positively charged jellium density $n_{+}(\mathbf{r})$ and electron density $n_{-}(\mathbf{r})$. The Hamiltonian of the system can be written as the sum of the kinetic energy, the exchange-correlation energy, and the Coulomb energy: ${ }^{5}$

$$
H=\int d^{3} r\left(E_{\mathrm{kin}}+E_{\mathrm{xc}}+E_{\mathrm{Coul}}\right),
$$

where

$$
\begin{aligned}
E_{\mathrm{kin}}= & a_{1} n_{-}^{5 / 3}(\mathbf{r})+a_{2}\left[\left|\nabla n_{-}(\mathbf{r})\right|^{2} / n_{-}(\mathbf{r})\right], \\
E_{\mathrm{xc}}= & -n_{-}^{4 / 3}(\mathbf{r})\left\{a_{3}+a_{4} /\left[a_{5}+n_{-}^{1 / 3}(\mathbf{r})\right]\right\}, \\
E_{\mathrm{Coul}}= & \frac{1}{2}\left[n_{-}(\mathbf{r})-n_{+}(\mathbf{r})\right] \\
& \times \int d^{3} r^{\prime}\left[n_{-}\left(\mathbf{r}^{\prime}\right)-n_{+}\left(\mathbf{r}^{\prime}\right)\right] /\left|\mathbf{r}-\mathbf{r}^{\prime}\right|,
\end{aligned}
$$

and $a_{1}=2.8712, a_{2}=\frac{1}{72}, a_{3}=0.7386, a_{4}=0.05641$, and $a_{5}=0.7953$, where all quantities are expressed in atomic units (energy unit $=27.2 \mathrm{eV}$, charge unit $=1$ electronic charge, length unit $=0.529 \AA$ A). Since we would like to calculate the response of the jellium sphere to an external perturbation, it is natural to expand the Hamiltonian in terms of the deviations $\delta n_{-}(\mathbf{r})=n_{-}(\mathbf{r})-n_{-}^{0}(\mathbf{r})$ and $\delta n_{+}(\mathbf{r})=n_{+}(\mathbf{r})-n_{+}^{0}(\mathbf{r})$ from the unperturbed state:

$$
\begin{aligned}
H= & H^{(0)}\left[n_{-}^{0}(\mathbf{r}), n_{+}^{0}(\mathbf{r})\right] \\
& +\delta H^{(1)}\left[\delta n_{-}(\mathbf{r}), \delta n_{+}(\mathbf{r}), n_{-}^{0}(\mathbf{r}), n_{+}^{0}(\mathbf{r})\right] \\
& +\delta H^{(2)}\left[\delta n_{-}(\mathbf{r}), \delta n_{+}(\mathbf{r}), n_{-}^{0}(\mathbf{r}), n_{+}^{0}(\mathbf{r})\right],
\end{aligned}
$$

where $\delta H^{(1)}$ is a linear functional of $\delta n_{-}(\mathbf{r})$ and $\delta n_{+}(\mathbf{r})$ and $\delta H^{(2)}$ is a quadratic functional of $\delta n_{-}(\mathbf{r})$ and $\delta n_{+}(\mathbf{r})$.

In our present case the source causing the deviations $\delta n_{-}(\mathbf{r})$ and $\delta n_{+}(\mathbf{r})$ is a static electric field $\mathscr{E}_{0}$ whose interaction energy with the jellium sphere can be written as 


$$
\begin{aligned}
\delta H_{\mathrm{int}} & =\int d^{3} r\left[n_{-}(\mathbf{r})-n_{+}(\mathbf{r})\right] \mathscr{E}_{0} \cdot \mathbf{r} \\
& =\int d^{3} r\left[\delta n_{-}(\mathbf{r})-\delta n_{+}(\mathbf{r})\right] \mathscr{E}_{0} \cdot \mathbf{r},
\end{aligned}
$$

where the term involving $\int\left[n_{-}^{0}(\mathbf{r})-n_{+}^{0}(\mathbf{r})\right] \mathscr{E}_{0} \cdot \mathbf{r} d^{3} r$ is zero because $n_{-}^{0}(\mathbf{r})$ and $n_{+}^{0}(\mathbf{r})$ necessarily possess spherical symmetry, whereas $\mathscr{E}_{0} \cdot \mathbf{r}$ has dipole symmetry. From linear response theory it also follows that the induced density modulations $\delta n_{ \pm}(r)$ must have the form

$$
\delta n_{-}(\mathbf{r})=q(r) \cos \theta \mathscr{E}_{0}
$$

and

$$
\delta n_{+}(\mathbf{r})=p(r) \cos \theta \mathscr{E}_{0},
$$

where $\theta$ is the polar angle measured from the direction of $\mathscr{B}_{0}$. A direct consequence of $\delta n_{ \pm}$'s $\cos \theta$ symmetry is that $\delta H^{(1)}=0$ and therefore can be dropped from consideration.

Up to now we have not taken into consideration the energy associated with modulation of the jellium density apart from its Coulomb interaction. To properly account for this aspect of the problem, it is reasonable to assume that the elastic energy (beyond those associated with compressing a plasma) should play a dominant role since, after all, that is the part responsible for distinguishing a solid from a Coulomb gas. We can express this fact mathematically by first writing the jellium density modulation $\delta n_{+}(r)$ as a function of the jellium displacement field $\mathbf{u}(\mathbf{r})$,

$$
\delta n_{+}(\mathbf{r})=-n_{+}^{0}(\mathbf{r}) \nabla \cdot \mathbf{u}(\mathbf{r}),
$$

then stating that the form of $u(r)$ must be expressible in terms of the eigenfunctions of the static elastic operator. In Appendix $\mathbf{A}$ it is shown that $\mathbf{u}$ may be written as

$$
\mathbf{u}(\mathbf{r})=\sum_{n=1}^{\infty} Q_{n} \mathbf{s}_{n}(\mathbf{r}),
$$

where $s_{n}(\mathbf{r})$ belongs to a class of vibrational eigenfunctions ${ }^{6}$ for a sphere with dipolar angular dependences (due to the symmetry requirement of $\delta n_{+}$):

$$
\begin{aligned}
\mathbf{s}_{n}(\mathbf{r})= & \hat{\mathbf{r}}\left[j_{1}^{\prime}\left(k_{n}^{c} r\right)+2 A_{n} j_{1}\left(k_{n}^{s} r\right) /\left(k_{n}^{s} r\right)\right] \cos \theta \\
& -\hat{\boldsymbol{\theta}}\left\{A_{n}\left[k_{n}^{s} r j_{1}\left(k_{n}^{s} r\right)\right]^{\prime} / k_{n}^{s} r+j_{1}\left(k_{n}^{c} r\right) /\left(k_{n}^{c} r\right)\right\} \sin \theta .
\end{aligned}
$$

In Eq. (6b) $\hat{\mathbf{r}}$ and $\hat{\boldsymbol{\theta}}$ are unit vectors in the radial and polar angle direction, respectively, $k_{n}^{c(s)}=\omega_{n} / v_{c(s)}$ is the compressional (shear) wave vector for the $n$th vibrational mode with frequency $\omega_{n}, v_{c(s)}$ is the compressional (shear) wave speed, $j_{1}$ denotes spherical Bessel function of order $1,\left(1, A_{n}\right)$ is the eigenvector associated with $n$th eigenfrequency, and $n=1,2,3, \ldots$ indexes eigenfrequencies in ascending order. The elastic potential energy associated with jellium modulation can now be written as

$$
\delta H_{\text {elast }}=\frac{1}{2} \sum_{n} m_{n}^{e} \omega_{n}^{2} Q_{n}^{2},
$$

where $m_{n}^{e}$ is the effective mass for the $n$th eigenmode of vibration. The mathematics of calculating the eigenfrequency $\omega_{n}$ and the eigenvector $\left(1, A_{n}\right)$, together with the derivation of Eq. (7) and the formula for $m_{n}^{e}$, are detailed in Appendix A.

By collecting various terms, the total energy of an elastic jellium sphere in an electric field may now be expressed as

$$
H_{\text {tot }}=H^{(0)}\left[n_{-}^{0}(\mathbf{r}), n_{+}^{0}(\mathbf{r})\right]+\delta H,
$$

with

$$
\delta H=\delta H^{(2)}+\delta H_{\text {int }}+\delta H_{\text {elast }} .
$$

To get the electronic and the jellium responses, we would like to minimize $H_{\text {tot }}$ with respect to $n_{-}(\mathbf{r})$ and $n_{+}(\mathbf{r})$. This task can be accomplished in two steps. First, one minimizes the energy of an isolated jellium sphere to get $n_{-}^{0}(\mathbf{r})$. We will assume that $n_{+}^{0}(\mathbf{r})$ is a constant equal to $\left(\frac{4}{3} \pi r_{s}^{3}\right)^{-1}$ for $r \leq R$ and zero for $r>R$, where $r_{s}$ is the electron density parameter. The unperturbed electron distribution $n_{-}^{0}(r)$ is then calculated variationally by using the trial function

$$
n_{-}^{0}(r)=\frac{c}{1+\exp [2 b(r-R-d)]+\exp [-2 b(r+R+d)]},
$$

suggested by Snider and Sorbello, ${ }^{2}$ with $b$ and $d$ as the variational parameters and $c$ being a normalization constant determined by the condition that the volume integral of $n_{-}^{0}(r)$ must be equal to $\left(R / r_{s}\right)^{3}$ for insuring electrical neutrality. Once the equilibrium configuration of an isolated sphere is obtained, the minimization of $\delta H$ [with respect to $\delta n_{-}(\mathbf{r})$ and $\left.\delta n_{+}(r)\right]$ may be reduced to a linear problem by expanding the radial part of $\delta n_{+}(\mathbf{r})$ and $\delta n_{-}(\mathbf{r})$ in terms of basis functions. For $\delta n_{+}(\mathbf{r})$, Eqs. (5) and (6) suggest that a natural basis set would be $\nabla \cdot \mathbf{s}_{n}(\mathbf{r})=-\left(\beta_{n} / R\right) \cos \theta j_{1}\left(\beta_{n} r / R\right)$ where $\beta_{n}=\omega_{n} R / v_{c}$ is the dimensionless eigenvalue of the $n$th vibrational mode. From Eqs. (4b) and (5), that means

$$
p(r)=\sum_{n} p_{n} j_{1}\left(\beta_{n} r / R\right),
$$

where the expansion coefficient $p_{n}$ is related to the amplitude $Q_{n}$ of the $n$th elastic normal mode by the relation

$$
Q_{n}=\frac{\mathscr{C}_{0} R}{n_{+}^{0} \beta_{n}} p_{n} .
$$

For $\delta n_{-}(\mathbf{r})$, we note that since the electron density perturbation must vanish at the center of the sphere (from dipole symmetry) and become exponentially small at some suitable distance (i.e., 8 a.u.) out from the sphere boundary, it is natural to expand $q(r)$ as

$$
q(r)=\sum_{m} q_{m} j_{1}\left(\alpha_{m} r / a\right)
$$

where $a=R+8$ a.u. is the outer radius limit for the definition of $q(r)$, and $\alpha_{n}$ 's denote the zeroes of $j_{1}$. Substitution of Eqs. (4), (10), and (11) into $\delta H^{(2)}, \delta H_{\text {int }}$, and $\delta H_{\text {elast }}$ yields the total perturbation energy as 


$$
\begin{aligned}
\delta H= & \delta H^{(2)}+\delta H_{\mathrm{int}}+\delta H_{\mathrm{elast}} \\
= & \left(\frac{1}{2} \sum_{i, j}\left(M_{i j} a^{3}+C_{i j} a^{5}\right) q_{i} q_{j}-\sum_{i, j}\left(D_{i j} a^{5}\right) q_{i} p_{j}\right. \\
& \left.+\frac{1}{2} \sum_{i, j}\left(E_{i j} a^{5}\right)+\delta_{i j}\left(m_{i}^{e} \lambda\right) /\left[\left(n_{+}^{0}\right)^{2} \rho\right]\right) p_{i} p_{j} \\
& \left.+\sum_{i}\left(F_{i} a^{4}\right) q_{i}-\sum_{i}\left(G_{i} a^{4}\right) p_{i}\right] \mathscr{E}_{0}^{2},
\end{aligned}
$$

where the matrix element $M_{i j}$ is derived from the electron kinetic and exchange-correlation energies, $C_{i j}$ is from the Coulomb repulsion between the electron densities, $D_{i j}$ from the attractive interaction between the electron and the jellium, $E_{i j}$ represents the Coulomb repulsion between the jellium densities, $\delta_{i j}\left(m_{i}^{e} \lambda\right) /\left[\left(n_{+}^{0}\right)^{2} \rho\right]$ is from the elastic jellium deformation energy with $\lambda$ being the compressional elastic modulus of the sphere, $F_{i}$ represents the interaction between the electron density and the electric field, and $G_{i}$ represents the interaction between the jellium and the electric field. Mathematical formulas for all the above-defined matrix elements, plus the derivation of the particular form for the elastic energy term, are presented in Appendix B. Given Eq. (12), the minimization of $\delta H$ is represented by the conditions

$$
\frac{\partial(\delta H)}{\partial q_{i}}=0
$$

and

$$
\frac{\partial(\delta H)}{\partial p_{i}}=0 .
$$

Written out with all the pertinent terms, these conditions are linear simultaneous equations for the determination of $q_{i}$ and $p_{i}$ :

$$
\begin{aligned}
& \sum_{j}\left(M_{i j} a^{-1}+C_{i j} a\right) q_{j}-\left(D_{i j} a\right) p_{j}=-F_{i} \\
& \sum_{j}-\left(D_{j i} a\right) q_{j}+\left(E_{i j} a+\delta_{i j} \frac{m_{i}^{e} \lambda}{a^{4} \rho\left(n_{+}^{0}\right)^{2}}\right) p_{j}=G_{i}
\end{aligned}
$$

Substitution of the resulting $q_{i}$ and $p_{i}$ into Eqs. (11), (10), and (4) completes the calculation for the response functions $\delta n_{-}(\mathbf{r})$ and $\delta n_{+}(\mathbf{r})$.

From $\delta n_{-}(\mathbf{r})$ and $\delta n_{+}(\mathbf{r})$, there are two ways for calculating the polarizability. One is to note that $\delta H$ is the energy associated with polarizing the sphere and therefore

$$
\delta H=-\frac{\alpha_{0}}{2} \mathscr{E}_{0}^{2}
$$

where $\alpha_{0}$ is the polarizability. Another approach involves the calculation of the net dipole moment

$$
p=\int r \cos \theta\left[\delta n_{-}(\mathbf{r})-\delta n_{+}(\mathbf{r})\right] d^{3} r=\alpha_{0} \mathscr{E}_{0}
$$

By using Eq. (13), it can be shown that the two methods are exactly equivalent and the resulting $\alpha_{0}$ is expressible as

$$
\begin{aligned}
& \alpha_{0}=\alpha_{0}^{e}-\alpha_{0}^{i}, \\
& \alpha_{0}^{e}=-\sum_{i} a^{4} q_{i} F_{i}=\mathscr{E}_{0}^{-1} \int r \cos \theta \delta n_{-}(\mathbf{r}) d^{3} r, \\
& \alpha_{0}^{i}=-\sum_{i} a^{4} p_{i} G_{i}=\mathscr{E}_{0}^{-1} \int r \cos \theta \delta n_{+}(\mathbf{r}) d^{3} r,
\end{aligned}
$$

where $\alpha_{0}^{e}$ is the electronic polarizability and $\alpha_{0}^{i}$ is the ionic polarizability.

One can also calculate the electric field profile inside and outside the sphere. The net Coulomb potential experienced by the electron and jellium densities due to the applied field and induced charges may be written as

$$
V(r, \theta)=-r \mathscr{E}_{0} \cos \theta-v(r) \mathscr{E}_{0} \cos \theta,
$$

where

$$
\begin{array}{r}
v(r)=\frac{4 \pi}{3}\left[\frac{1}{r^{2}} \int_{0}^{r}\left(r^{\prime}\right)^{3}\left[q\left(r^{\prime}\right)-p\left(r^{\prime}\right)\right] d r^{\prime}\right. \\
\left.+r \int_{r}^{\infty}\left[q\left(r^{\prime}\right)-p\left(r^{\prime}\right)\right] d r^{\prime}\right]
\end{array}
$$

is the potential generated by the screening charges. The net electric field (in the direction of the applied field) is then given by

$$
\mathscr{E}=\mathscr{E}_{0}\left(1+\frac{d v(r)}{d r}\right) .
$$

\section{RESULTS AND DISCUSSION}

The formulation developed above was used to calculate the response of a small metal sphere in a static electric field. For the parameter $r_{s}$, we used a value of 3.93 a.u. corresponding to the electron density ${ }^{7}$ of bulk Na. For the elastic properties of the particle we have employed a value of 1.7 for the ratio between the compressional wave speed and the shear wave speed, and $\lambda$ was treated as a variable with the bulk modulus ${ }^{8}$ of $\mathrm{Na}, \lambda_{0}=0.068 \times 10^{12}$ dynes $/ \mathrm{cm}^{2}$, serving as the reference unit. Since all the input parameters except $\lambda$ were picked to have $\mathrm{Na}$ values, it follows that the jellium sphere in consideration should approximate a $\mathrm{Na}$ cluster when $\lambda \simeq \lambda_{0}$.

After the equilibrium density function $n_{-}^{0}(r)$ [Eq. (9)] was calculated variationally, the jellium and the electronic density modulations were obtained by solving Eqs. (13a) and (13b). Convergence of the final results was checked by varying the number of basis functions used to expand $p(r)$ and $q(r)$ [Eqs. (10) and (11)]. It was found that 30 elastic eigenfunctions and 30 electronic basis functions are generally sufficient for resolving the details of the electronic and ionic modulations. In Table I we display the polarizability values (normalized to $R^{3}=N r_{s}^{3}$ ) for nine cluster sizes $(N=8,18,20,34,40,58,68,70,92)$ corresponding to those possessing closed electronic shells. ${ }^{3}$ It is seen that as the elastic modulus $\lambda$ of the sphere is varied the particle polarizability (given by the difference between the electronic and the ionic components) rises at most $1-2 \%$ from its rigid-core value (which corresponds closely to that calculated by the self-consistent scheme $^{2}$ ). 
TABLE I. Normalized particle polarizability $\alpha_{0}$ and ionic polarizability $\alpha_{0}^{i}$ (in parentheses) for nine different cluster sizes and seven different elastic stiffness constant values. The unit of polarizability is $R^{3} \equiv N r_{s}^{3} . \quad\left(\lambda_{0}=0.068 \times 10^{12}\right.$ dynes $\left./ \mathrm{cm}^{2}.\right)$

\begin{tabular}{cccccccc}
\hline \hline & & & & & $\lambda_{0} / \lambda$ \\
$N$ & 0 & 1 & 2 & 4 & 8 & 16 & 32 \\
\hline 8 & (rigid) & & & & & & \\
& 1.449 & 1.461 & 1.468 & 1.476 & 1.484 & 1.491 & 1.495 \\
18 & & $(0.172)$ & $(0.281)$ & $(0.412)$ & $(0.539)$ & $(0.639)$ & $(0.706)$ \\
& 1.311 & 1.318 & 1.323 & 1.328 & 1.332 & 1.336 & 1.338 \\
20 & & $(0.170)$ & $(0.274)$ & $(0.395)$ & $(0.510)$ & $(0.597)$ & $(0.654)$ \\
& 1.299 & 1.306 & 1.310 & 1.315 & 1.319 & 1.322 & 1.324 \\
34 & & $(0.170)$ & $(0.274)$ & $(0.395)$ & $(0.508)$ & $(0.595)$ & $(0.651)$ \\
& 1.247 & 1.252 & 1.255 & 1.258 & 1.262 & 1.264 & 1.266 \\
40 & & $(0.171)$ & $(0.274)$ & $(0.392)$ & $(0.500)$ & $(0.582)$ & $(0.635)$ \\
& 1.232 & 1.237 & 1.240 & 1.243 & 1.246 & 1.248 & 1.250 \\
58 & & $(0.171)$ & $(0.273)$ & $(0.390)$ & $(0.497)$ & $(0.577)$ & $(0.628)$ \\
& 1.204 & 1.208 & 1.210 & 1.213 & 1.215 & 1.217 & 1.218 \\
68 & & $(0.171)$ & $(0.271)$ & $(0.385)$ & $(0.488)$ & $(0.564)$ & $(0.613)$ \\
& 1.192 & 1.196 & 1.198 & 1.200 & 1.202 & 1.204 & 1.205 \\
70 & & $(0.170)$ & $(0.270)$ & $(0.382)$ & $(0.483)$ & $(0.557)$ & $(0.605)$ \\
& 1.190 & 1.193 & 1.196 & 1.198 & 1.200 & 1.202 & 1.203 \\
92 & & $(0.170)$ & $(0.270)$ & $(0.381)$ & $(0.482)$ & $(0.556)$ & $(0.603)$ \\
& 1.172 & 1.175 & 1.177 & 1.179 & 1.181 & 1.183 & 1.183 \\
& & $(0.169)$ & $(0.267)$ & $(0.376)$ & $(0.473)$ & $(0.504)$ & $(0.589)$ \\
\hline \hline
\end{tabular}

However, the ionic polarizability, given in the parentheses, is seen to increases as $\lambda$ decreases. Even at $\lambda=\lambda_{0}(\mathrm{Na}$ clusters), the ionic component is about $11-14 \%$ of the particle polarizability. In Fig. 1, the continuous variation of the ionic polarizability with elastic stiffness constant $\lambda$ is depicted graphically. For large $\lambda$ (small $\lambda_{0} / \lambda$ ), the variation can be approximately described by the relation

$$
\alpha_{0}^{i} / R^{3} \simeq 0.2 \operatorname{arcsinh}\left(\lambda_{0} / \lambda\right)
$$

for all the sphere sizes considered. However, in the limit of small $\lambda$, the increase in $\alpha_{0}^{i}$ becomes slower than that

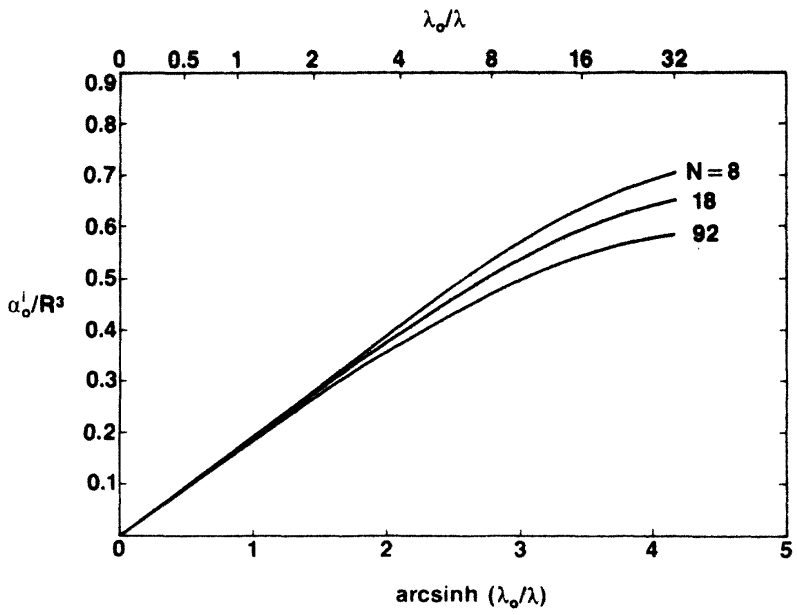

FIG. 1. Normalized ionic polarizability as a function of $\operatorname{arcsinh}\left(\lambda_{0} / \lambda\right)$ for three different cluster sizes. given by Eq. (19). While not plotted, the variation of the electronic polarizability $\alpha_{0}^{e}$ is expected to be similar as those shown in Fig. 1 since the net particle polarizability is relatively constant as a function of $\lambda$.

The presence of a non-negligible ionic component of polarizability is reflected most clearly in the charge density modulations. In Fig. 2 the radial parts of the electron and jellium modulations, $q(r)$ and $p(r)$, are plotted as a function of $r / R$ for an 18 particle $\mathrm{Na}$ cluster. The peak of the jellium modulation is seen to be $\sim 30 \%$ that of the electronic modulation. Also, there is a discontinuous drop

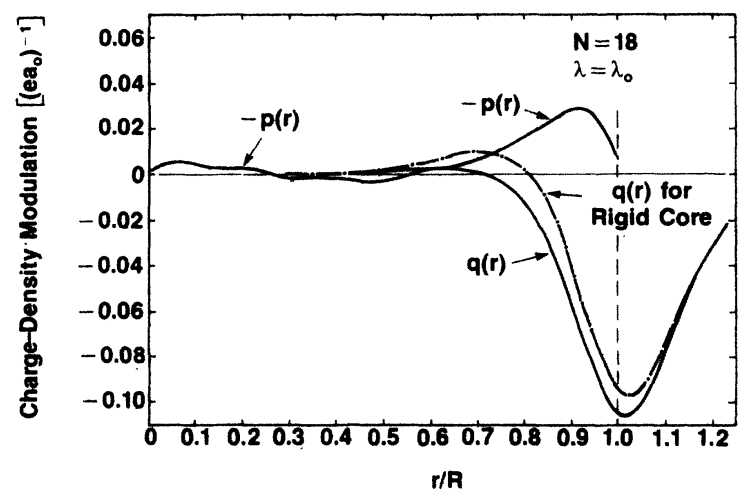

FIG. 2. Electron and ionic jellium density modulations for an 18-particle $\mathrm{Na}$ cluster. The electron modulation profile for the rigid jellium model is also plotted for comparison. The unit of charge density modulation is $\left(e a_{0}\right)^{-1}$, where $e$ is the electronic charge and $a_{0}=0.529 \AA$. 


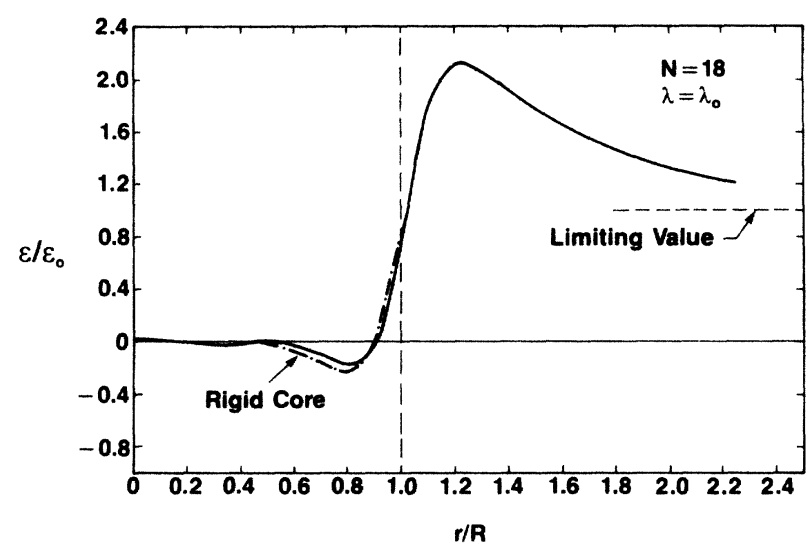

FIG. 3. Electric field profile for the 18-particle $\mathrm{Na}$ cluster. The rigid core case is also plotted for comparison.

in the value of $-p(r)$ (to zero) just outside the sphere boundary because the jellium does not extend beyond $r=R$.

The electric field profile for the same cluster is shown in Fig. 3. Compared to the rigid-core case, here the effect of jellium elasticity is only to slightly decrease the penetration of the field into the sphere interior, while outside the sphere the electric field for the elastic sphere is indistinguishable from that of the rigid core sphere. In both cases the value of $\mathscr{E}$ displays a peak with a magnitude of $\sim 2 \mathscr{E}_{0}$ just outside the sphere and subsequently decays towards the limiting value of $\mathscr{E}_{0}$ far away from the sphere boundary.

\section{CONCLUDING REMARKS}

In this work we have shown that the relaxation of jellium rigidity leads to a significant departure from the rigid-core model in both the ionic and/or electronic makeup of the polarizability and the accompanying charge density modulations. In relating these jellium model results to actual metal clusters, two points concerning the approximations of the theory need to be addressed. First, the present theory extends the jellium continuum picture to the treatment of the elastic property. While the smearing of the ionic background has been shown by previous works to be a reasonably good approximation with respect to the electron-ion interaction, its accuracy in regard to the elastic aspect of the problem is not as well assessed. However, from the experience of the effective medium theory it is known that in approximating a discrete or inhomogeneous system by an effective homogeneous continuum, the accuracy improves as the wavelength of distortion becomes larger than the scale of inhomogeneities. Therefore, on relative terms we expect our results to be more realistic for the larger clusters than for the smaller ones. But in all cases it should be noted that compared to the rigid jellium case, the elastic jellium is already a more realistic model. The second point we would like to remark is that the simple form of the density functional theory used in our calculation precludes shell structure effects. This was done because the shell structure is not the aim of this work. Instead, we have concentrated on the calculation of elasticity effects on a restricted number of cluster sizes, i.e., those with closed shell numbers for which the rigid-core polarizability has been calculated by the more sophisticated density functional theory. The fact that our numbers in the rigid-core limit calibrate favorably with those calculated by other methods ${ }^{2}$ (as noted earlier in the test) thus provides a validation for our usage of the simpler density functional theory.

In conclusion, we have considered the elastic effects of the jellium model and found a non-negligible elastic component in the polarizing response of a jellium sphere. As one of the first attempts in this direction, it is our hope that the above discussion will stimulate further work on the elastic aspect of the small cluster behavior.

\section{ACKNOWLEDGMENTS}

Two of us (M.Y.C. and M.L.C.) are supported by the National Science Foundation under Grant No. DMR 8319024 and by the Director, Office of Energy Research, Office of Basic Energy Sciences, Material Sciences Division of the U.S. Department of Energy under Contract No. DE-AC03-76SF00098.

\section{APPENDIX A}

Here we present the mathematical details relevant to the elastic aspect of the problem. Since the calculation of jellium modulation is perturbative in nature, we would like to expand the ionic jellium distortion field $\mathbf{u}(\mathbf{r})$ in terms of the eigenfunctions of the static elastic operator

$$
\mathscr{L}=\lambda \operatorname{grad} \operatorname{div}-\mu \text { curl curl }
$$

for an unperturbed sphere. Here $\lambda$ is the compressional modulus and $\mu$ is the shear modulus of the sphere. The eigenvalue equation of

$$
\mathscr{L} \mathbf{u}=-\Gamma^{2} \mathbf{u}
$$

is easily recognized as the frequency transform of the elastic wave equation if one writes $\Gamma^{2}=\omega^{2} \rho$, where $\omega$ is the angular frequency and $\rho$ the density. Therefore the eigenfunctions of the operator $\mathscr{L}$ are equivalent to the solutions of the elastic vibrational problem.

In general, there are three independent types of solutions $^{9}$ to Eq. (A2):

$$
\begin{aligned}
& \mathbf{u}=\mathbf{L}_{m l} \equiv(1 / k) \operatorname{grad}\left[X_{m l}(\theta, \phi) j_{l}(k r)\right], \\
& k^{2}=\Gamma^{2} / \lambda=\omega^{2} \rho / \lambda \\
& \mathbf{u}=\mathbf{M}_{m l} \equiv \operatorname{curl}\left[\mathbf{r} X_{m l}(\theta, \phi) j_{l}(k r)\right], \quad k^{2}=\Gamma^{2} / \mu=\omega^{2} \rho / \mu
\end{aligned}
$$

and

$$
\begin{aligned}
& \mathbf{u}=\mathbf{N}_{m l} \equiv(1 / k) \operatorname{curl} \operatorname{curl}\left[\mathbf{r} X_{m l}(\theta, \phi) j_{l}(k r)\right] \\
& k^{2}=\Gamma^{2} / \mu=\omega^{2} \rho / \mu .
\end{aligned}
$$

The notation $X_{m l}(\theta, \phi)$ here denotes the spherical harmonics of order $(m, l), \theta, \phi$ are the polar and the azimuthal angles, respectively, and the symbol $j_{l}$ has the same meaning 
as that defined in the text. From the $\omega-k$ relations of the three types of eigenfunctions, it is easy to deduce that $\mathbf{L}_{m l}$ corresponds to a compressional distortion whereas $\mathbf{M}_{m l}$ and $\mathbf{N}_{m l}$ represent shear distortions.

For an unperturbed sphere, we have the boundary condition that the surface of the sphere is traction (force) free. Analysis shows that such a condition can be satisfied by $\mathbf{M}_{m l}$ alone, or by linear combinations of $\mathbf{L}_{m l}$ and $\mathbf{N}_{m l}$, i.e.,

$$
\mathbf{u}=\mathbf{L}_{m l}(\mathbf{r})+A \mathbf{N}_{m l}(\mathbf{r}),
$$

where $A$ is a constant to be adjusted. Since the modes represented by $\mathbf{M}_{m l}$ are purely torsional and therefore do not couple to jellium modulations $\left(\boldsymbol{\nabla} \cdot \mathbf{M}_{m l}=0\right)$, they will be dropped from further consideration. For a displacement field of the form expressed by Eq. (A3), the traction-free condition at $r=R$ yields the following $2 \times 2$ matrix equation: ${ }^{6}$

$$
\left.\left[\begin{array}{l}
0 \\
0
\end{array}\right]=\left(\begin{array}{cr}
2 j_{l}^{\prime \prime}\left(k_{c} R\right)-\left[\left(\frac{k_{s}}{k_{c}}\right]^{2}-2\right] j_{l}\left(k_{c} R\right) & \frac{k_{s}}{k_{c}}[2 l(l+1)]\left(\frac{j_{l}(x)}{x}\right)_{x=k_{s} R}^{\prime} \\
2 j_{l}^{\prime}\left(k_{c} R\right)-\frac{2}{k_{c} R} j_{l}\left(k_{c} R\right) & k_{s} R\left(j_{l}^{\prime \prime}\left(k_{s} R\right)+\frac{\left(l^{2}+l-2\right)}{\left(k_{s} R\right)^{2}} j_{l}\left(k_{s} R\right)\right.
\end{array}\right]\right)\left[\begin{array}{l}
1 \\
A
\end{array}\right],
$$

where $k_{c}=\omega(\rho / \lambda)^{1 / 2}$ and $k_{s}=\omega(\rho / \mu)^{1 / 2}$. For a given ratio $\kappa=k_{s} / k_{c}=(\lambda / \mu)^{1 / 2}$, Eq. (A4) expresses a condition for the determination of the dimensionless eigenvalue $\beta=k_{c} R$ and the corresponding eigenvector $(1, A)$. Since we are only interested in those distortions possessing dipole symmetry, the relevant eigenvalue equation is obtained by letting $l=1$, taking the determinant of the matrix in Eq. (A4), and setting it equal to zero:

$$
\begin{aligned}
0= & \beta\left[2 j_{1}^{\prime \prime}(\beta)-\left(\kappa^{2}-2\right) j_{1}(\beta)\right] j_{1}^{\prime \prime}(\kappa \beta) \\
& -\frac{8}{\kappa \beta}\left[j_{1}(\beta)-\frac{1}{\beta} j_{1}(\beta)\right]\left[j_{1}^{\prime}(\kappa \beta)-\frac{1}{\kappa \beta} j_{1}(\kappa \beta)\right] .
\end{aligned}
$$

If we index the solution of Eq. (A5) in ascending order as $\beta_{n}, n=1,2,3, \ldots$, then the $A_{n}$ of the associated eigenvector $\left(1, A_{n}\right)$ is given by

$$
A_{n}=\kappa \beta_{n} \frac{\left(\kappa^{2}-2\right) j_{1}\left(\beta_{n}\right)-2 j_{1}^{\prime \prime}\left(\beta_{n}\right)}{4\left[j_{1}^{\prime}\left(\kappa \beta_{n}\right)-\frac{1}{\kappa \beta_{n}} j_{1}\left(\kappa \beta_{n}\right)\right]}
$$

Written out explicitly, the (vibrational) eigenfunction $\mathbf{s}_{n}(\mathbf{r})$ has the form expressed by $\mathrm{Eq}$. (6b). The potential energy of the distortion associated with the displacement field $Q_{n} s_{n}(r)$ can now be written as

$$
\begin{aligned}
V_{\text {pot }} & =\frac{1}{2} \int(\text { displacement }) \times(\text { force/unit volume }) d v \\
& =\frac{1}{2} Q_{n}^{2} \int_{\text {sphere }} \mathbf{s}_{n} \cdot\left(\mathscr{L} \mathbf{s}_{n}\right) d v \\
& =\frac{1}{2} \rho \omega_{n}^{2} Q_{n}^{2} \int_{\text {sphere }} \mathbf{s}_{n} \cdot \mathbf{s}_{n} d v=\frac{1}{2} m_{n}^{e} \omega_{n}^{2} Q_{n}^{2},
\end{aligned}
$$

where

$$
\omega_{n}=\frac{1}{R}\left(\frac{\lambda}{\rho}\right)^{1 / 2} \beta_{n}
$$

is the eigenfrequency of the $n$th vibrational mode,

$$
m_{n}^{e}=\frac{4 \pi}{3} R^{3} \rho \frac{c_{n}}{\beta_{n}^{2}}
$$

is the effective mass involved in the vibration, and the constant $c_{n}$ denotes the following integral:

$$
\begin{gathered}
c_{n}=\int_{0}^{1} d x\left[\left[-2 j_{1}\left(\beta_{n} x\right)+2 \frac{A_{n}}{\kappa} j_{1}\left(\kappa \beta_{n} x\right)+\sin \left(\beta_{n} x\right)\right]^{2}\right. \\
+2\left[j_{1}\left(\beta_{n} x\right)-\frac{A_{n}}{\kappa} j_{1}\left(\kappa \beta_{n} x\right)\right. \\
\left.\left.+\frac{A_{n}}{\kappa} \sin \left(\kappa \beta_{n} x\right)\right]^{2}\right]
\end{gathered}
$$

Combining Eqs. (A7), (A8), and (A9) yields

$$
V_{\text {pot }}=\frac{2 \pi}{3} N^{1 / 3} r_{s} \lambda c_{n} Q_{n}^{2},
$$

where we have substituted $N^{1 / 3} r_{s}$ for $R$. For a distortion field $u$ expanded in terms of $s_{n}$ as

$$
\mathbf{u}=\sum_{n} Q_{n} \mathbf{s}_{n},
$$

the total elastic energy is obtained by superposing the energies of the different eigenmodes:

$$
E_{\text {elast }}^{\text {tot }}=\frac{2 \pi}{3} N^{1 / 3} r_{s} \lambda \sum_{n} c_{n} Q_{n}^{2} .
$$

To express Eq. (A13) in terms of the expansion parameters $p_{n}$ for $\delta n_{+}(r)$, we use Eq. (10b). This results in

$$
E_{\text {elast }}^{\text {tot }}=\frac{2 \pi}{3} N r_{s}^{3} \lambda \frac{\mathscr{E}_{0}^{2}}{\left(n_{+}^{0}\right)^{2}} \sum_{n} \frac{c_{n}}{\beta_{n}^{2}} p_{n}^{2} \text {. }
$$

\section{APPENDIX B}

The matrix elements for $\delta H$ are obtained by substituting the expansion forms of $p(r)$ and $q(r)$ [Eqs. (10) and (11)] into the expressions for $\delta H^{(2)}, \delta H_{\text {int }}$, and $\delta H_{\text {elast }}$ and by performing the necessary integrals, whenever possible, over the basic functions. Following is a tabulation of results: 


$$
\begin{aligned}
& M_{i j}=\frac{4 \pi}{3} \int_{0}^{1} x^{2} d x\left[2 N_{0}(a x) j_{1}\left(\alpha_{i} x\right) j_{1}\left(\alpha_{j} x\right)+2 N_{1}(a x) \frac{\alpha_{i} \alpha_{j}}{a^{2}} j_{1}^{\prime}\left(\alpha_{i} x\right) j_{1}^{\prime}\left(\alpha_{j} x\right)\right. \\
& \left.+N_{2}(a x)\left[\frac{\alpha_{i}}{a} j_{1}^{\prime}\left(\alpha_{i} x\right) j_{1}\left(\alpha_{j} x\right)+\frac{\alpha_{j}}{a} j_{1}^{\prime}\left(\alpha_{j} x\right) j_{1}\left(\alpha_{i} x\right)\right]\right) \\
& N_{0}(r)=\frac{5}{9} a_{1}\left(n_{-}^{0}\right)^{-1 / 3}+a_{2}\left(n_{-}^{0}\right)^{-3}\left(\frac{\partial n_{-}^{0}}{\partial r}\right)^{2}-\frac{1}{9}\left(n_{-}^{0}\right)^{-2 / 3}\left(2 a_{3}+a_{4} a_{5} \frac{2 a_{5}+\left(n_{-}^{0}\right)^{1 / 3}}{\left[a_{5}+\left(n_{-}^{0}\right)^{1 / 3}\right]^{3}}\right)+\frac{2 a_{2}}{n_{-}^{0} r^{2}}, \\
& N_{1}(r)=\frac{a_{2}}{n_{-}^{0}}, \\
& N_{2}(r)=-2 a_{2}\left(n_{-}^{0}\right)^{-2} \frac{\partial n_{-}^{0}}{\partial r}
\end{aligned}
$$

where $n_{-}^{0}$ denotes $n_{-}^{0}(r), a_{1}, a_{2}, a_{3}, a_{4}$, and $a_{5}$ are constants already defined in the text, and $\alpha_{i}$ denotes zero of $j_{1}(\alpha)$. The Coulomb repulsion energy between electrons is given by

$$
\begin{aligned}
& C_{i j}=\frac{8 \pi^{2}}{9}\left[\frac{f\left(\alpha_{i}\right)}{\alpha_{j}^{2} \alpha_{i}^{4}}\left[\frac{3}{2 \alpha_{i}^{2}} f\left(\alpha_{i}\right) \delta_{i j}-\sin \alpha_{j}\right]+\frac{f\left(\alpha_{j}\right)}{\alpha_{i}^{2} \alpha_{j}^{4}}\left[\frac{3}{2 \alpha_{j}^{2}} f\left(\alpha_{j}\right) \delta_{i j}-\sin \alpha_{i}\right]\right], \\
& f(\alpha)=\left(3-\alpha^{2}\right) \sin \alpha-3 \alpha \cos \alpha .
\end{aligned}
$$

Jellium-electron interaction energy is contained in

$$
\begin{aligned}
D_{i j}=\frac{16 \pi^{2}}{9}\left[\frac{R}{a}\right]^{3} \frac{1}{\left(\alpha_{i} \beta_{j}\right)^{2}} & \left\{\frac{3\left(\beta_{j} a\right)^{2}}{\left(\alpha_{i} R\right)^{2}-\left(\beta_{j} a\right)^{2}}\left[\sin \beta_{j} j_{1}\left[\alpha_{i} \frac{R}{a}\right)-\sin \left[\alpha_{i} \frac{R}{a}\right) j_{1}\left(\beta_{j}\right)\right]\right. \\
& \left.-\left(\frac{R}{a}\right) \sin \alpha_{i}\left[3 j_{1}\left(\beta_{j}\right)-\sin \beta_{j}\right]\right\} .
\end{aligned}
$$

Here $\beta_{j}$ denotes the elastic eigenvalues defined by the solution to Eq. (A5). Coulomb repulsion between ions yields

$$
\begin{aligned}
& E_{i j}=\frac{8 \pi^{2}}{9}\left[\frac{R}{a}\right]^{5}\left(h_{i j}+g_{i j}+h_{j i}+g_{j i}\right), \\
& h_{i j}=\left\{\begin{array}{l}
\frac{3}{\beta_{j}^{2}} \frac{\sin \beta_{j} j_{1}\left(\beta_{i}\right)-\sin \beta_{i} j_{1}\left(\beta_{j}\right)}{\left(\beta_{i}^{2}-\beta_{j}^{2}\right)}, i \neq j \\
\frac{3}{\beta_{i}^{5}}\left[\frac{\beta_{i}+\cos \beta_{i} \sin \beta_{i}}{2}-\frac{\sin ^{2} \beta_{i}}{\beta_{i}}\right], i=j
\end{array}\right. \\
& g_{i j}=-\frac{\sin \beta_{j}}{\beta_{j}^{2} \beta_{i}^{4}} f\left(\beta_{i}\right)
\end{aligned}
$$

where the function $f$ is defined by Eq. (B6). The elastic energy matrix element can be obtained directly from $\mathrm{Eq}$. (A14) by noting that $m_{n}^{e}=(4 \pi / 3) N r_{s}^{3} \rho c_{n} / \beta_{n}^{2}$. Finally, the vector elements $F_{i}$ and $G_{i}$ expressing the interaction energy of the electric field with, respectively, the electronic and the jellium densities can be written as

$$
\begin{aligned}
F_{i} & =\frac{1}{\alpha_{i}^{4}} \frac{4 \pi}{3} f\left(\alpha_{i}\right), \\
G_{i} & =\left(\frac{R}{a}\right)^{4} \frac{1}{\beta_{i}^{4}} \frac{4 \pi}{3} f\left(\beta_{i}\right) .
\end{aligned}
$$

*Present address: Corporate Research Science Laboratories, Exxon Research and Engineering Company, Clinton Township, Annandale, NJ 08801.

'W. D. Knight, K. Clemenger, W. A. deHeer, and W. A. Saunders, Phys. Rev. B 31, 2539 (1985).

2W. Ekardt, Phys. Rev. Lett. 52, 1925 (1984); D. R. Snider and R. S. Sorbello, Phys. Rev. B 28, 5702 (1983); D. E. Beck, ibid. 30, 6935 (1984); M. J. Puska, R. M. Nieminen, and M. Manninen, ibid. 31, 3486 (1985).

${ }^{3}$ W. D. Knight, K. Clemenger, W. A. DeHeer, W. A. Saunders, M. Y. Chou, and M. L. Cohen, Phys. Rev. Lett. 52, 2141 (1984).
${ }^{4}$ P. Sheng, Phys. Rev. B 31, 4906 (1985).

${ }^{5} \mathrm{~W}$. Kohn and P. Vashishta, in Theory of the Inhomogeneous Electron Gas, edited by S. Lundqvist and N. H. March (Plenum, New York, 1983), p. 75.

6P. M. Morse and H. Feshbach, Methods of Theoretical Physics (McGraw-Hill, New York, 1953), Vol. II, p. 1759.

${ }^{7}$ C. Kittel, Introduction to Solid State Physics, 5th Ed. (Wiley, New York, 1976), p. 154.

${ }^{8}$ C. Kittel, Introduction to Solid State Physics, 5th Ed., Ref. 7, p. 85. It should be noted that the compressional modulus $\lambda$ is actually equal to $k+(4 / 3) \mu$, where $k$ is the bulk modulus. However, we use the bulk modulus value as an approximation 
for the compressional modulus of $\mathrm{Na}$ because (i) the elastic properties of small particles are not known accurately but could be expected to deviate from that of the bulk, and (ii) the compressional modulus $\lambda$ used in our calculation should have the Coulombic part excluded since that part of the elastic energy is already being taken into account separately through the matrix elements $D_{i j}$ and $E_{i j}$. The value of $\lambda_{0}$ therefore should be viewed only as a rough reference probably within a factor of $\sim 2$ of the true value.

${ }^{9}$ P. M. Morse and H. Feshbach, Methods of Theoretical Physics, Ref. 6, p. 1865. 